\title{
Organization of Energetic Particles by the Solar Wind Structure During the Declining to Minimum Phase of Solar Cycle 23
}

\author{
C.O. Lee · J.G. Luhmann • I. de Pater • G.M. Mason • \\ D. Haggerty • I.G. Richardson • H.V. Cane · L.K. Jian • \\ C.T. Russell • M.I. Desai
}

Received: 27 December 2009 / Accepted: 5 April 2010 / Published online: 21 April 2010

(C) The Author(s) 2010. This article is published with open access at Springerlink.com

\begin{abstract}
We investigate the organization of the low energy energetic particles $(\leq 1 \mathrm{MeV})$ by solar wind structures, in particular corotating interaction regions (CIRs) and shocks driven by interplanetary coronal mass ejections, during the declining-to-minimum phase of Solar Cycle 23 from Carrington rotation 1999 to 2088 (January 2003 to October 2009). Because CIR-associated particles are very prominent during the solar minimum, the unusually long solar minimum period of this current cycle provides an opportunity to examine the overall organization of CIR energetic particles for a much longer period than during any other minimum since the dawn of the Space Age. We find that the particle enhancements associated
\end{abstract}

C.O. Lee $(\bowtie) \cdot$ J.G. Luhmann

Space Sciences Laboratory, University of California, Berkeley, CA, USA

e-mail: clee@ssl.berkeley.edu

C.O. Lee $\cdot$ I. de Pater

Department of Earth and Planetary Science, University of California, Berkeley, CA, USA

G.M. Mason · D. Haggerty

The Johns Hopkins University Applied Physics Laboratory, Laurel, MD, USA

I.G. Richardson - H.V. Cane

Goddard Space Flight Center, National Aeronautics and Space Administration, Greenbelt, MD, USA

I.G. Richardson

CRESST and Department of Astronomy, University of Maryland, College Park, MD, USA

H.V. Cane

School of Mathematics and Physics, University of Tasmania, Hobart, Tasmania, Australia

L.K. Jian · C.T. Russell

Institute of Geophysics and Planetary Physics, University of California, Los Angeles, CA, USA

M.I. Desai

Southwest Research Institute, San Antonio, TX, USA

M.I. Desai

Department of Physics and Astronomy, University of Texas, San Antonio, TX, USA 
with CIRs this minimum period recurred for many solar rotations, up to 30 at times, due to several high-speed solar wind streams that persisted. However, very few significant CIRrelated energetic particle enhancements were observed towards the end of our study period, reflecting the overall weak high-speed streams that occurred at this time. We also contrast the solar minimum observations with the declining phase when a number of solar energetic particle events occurred, producing a mixed particle population. In addition, we compare the observations from this minimum period with those from the previous solar cycle. One of the main differences we find is the shorter recurrence rate of the high-speed solar wind streams ( $\sim 10$ solar rotations) and the related CIR energetic particle enhancements for the Solar Cycle 22 minimum period. Overall our study provides insight into the coexistence of different populations of energetic particles, as well as an overview of the large-scale organization of the energetic particle populations approaching the beginning of Solar Cycle 24.

Keywords Energetic particles · Solar wind · Solar activity cycle $\cdot$ Magnetic fields: interplanetary

\section{Introduction}

Using data accumulated over the declining-to-minimum phase of Solar Cycle 23 (hereafter SC 23), we investigate the organization by the solar wind structure of the energetic ions (0.3 to $0.7 \mathrm{MeV})$ and electrons $(0.05$ to $0.1 \mathrm{MeV})$ from different particle populations. In particular, we focus on the energetic particles that are associated with corotating interaction regions (CIRs) and those associated with solar events. Depending on the phase of the solar cycle, these two populations of energetic particles can mix with each other at times. The heliospheric distribution of the energetic particles and its dependence on the solar cycle phase is of interest since it tells us about the big picture of particle acceleration in the vicinity of the Sun-like stars.

Figure 1 (top) illustrates a stream structure that corotates in the solar equatorial plane in the inner heliosphere. A CIR forms where a high-speed stream originating in a coronal hole runs into the slower speed stream that precedes it. In the declining speed region of the high-speed stream, a rarefaction region forms. As the coronal holes that give rise to the high-speed streams may persist for several solar rotations, the streams may be observed to recur one or more times at intervals of the solar rotation period $(\sim 27$ days as viewed from the Earth). Such recurring streams are very prominent during both the declining phase and the minimum period of the solar cycle (Richardson, 2004).

Shocks associated with CIRs can accelerate solar wind particles and produce energetic particles. Typically, these shocks form beyond Earth at one astronomical unit (AU) and accelerate energetic particles that stream into the inner heliosphere (see, e.g., Barnes and Simpson, 1976; van Hollebeke et al., 1978). However, CIR shocks can also form at and within 1 AU (Gosling and Pizzo, 1999; Forsyth and Marsch, 1999; Schwenn, 1978), and energetic particles associated with such CIRs may also be locally accelerated even in the absence of shocks. A typical intensity-time profile of CIR particles observed at $1 \mathrm{AU}$ is shown in Figure 1 (bottom panel). In this example, each shaded area in the profile indicate particle increases due to CIRs that appeared on four successive 27-day solar rotations. The figure shows that CIR-related particles are found in the high-speed stream, consistent with the particles being accelerated at the reverse shock and streaming to $1 \mathrm{AU}$ inside the highspeed stream. 

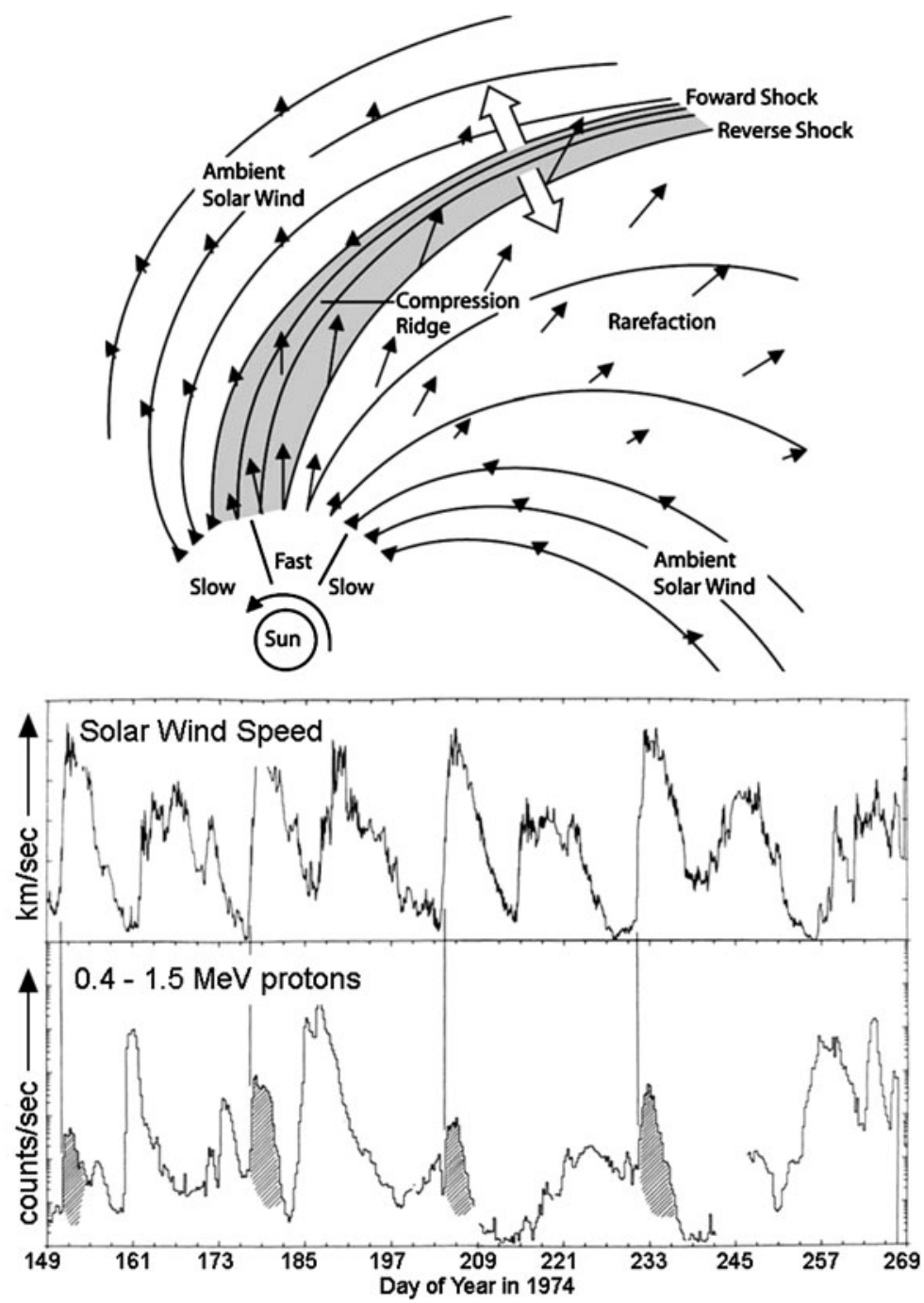

Figure 1 (Top) Illustration of a 2D stream structure in the solar equatorial plane of the inner heliosphere (adapted from Pizzo, 1978; Jian et al. 2006a, 2006b). (Bottom) Solar wind speed and energetic proton intensity observed by the IMP-8 spacecraft (adapted from Mason and Sanderson, 1999; Scholer et al., 1979). The shaded profiles indicate events associated with corotating high-speed streams whereas the unshaded profiles are related to solar events.

Figure 1 (bottom panel) also shows that between the successive CIR-related particle events, there are particle increases related to solar events, as shown in the unshaded portions. Solar activity such as impulsive flares and coronal mass ejections can accelerate particles and produce a particle population called solar energetic particles (SEPs). At the Sun, impulsive flares locally accelerate particles to higher energies. Such flare-accelerated SEPs can have very prompt signatures in the intensity - time profiles observed at $1 \mathrm{AU}$, as shown in Figure 2. These SEP intensities rise abruptly before settling back to the ambient level a couple of days later. 
Figure 2 Intensity-time profiles of energetic electrons ( 0.2 to $2 \mathrm{MeV}$ ) and protons (1 to $4 \mathrm{MeV}, 7$ to $13 \mathrm{MeV}$, and 22 to $27 \mathrm{MeV}$ ) associated with solar flare events, as measured by ISEE-3. Figure adapted from Reames (1999).

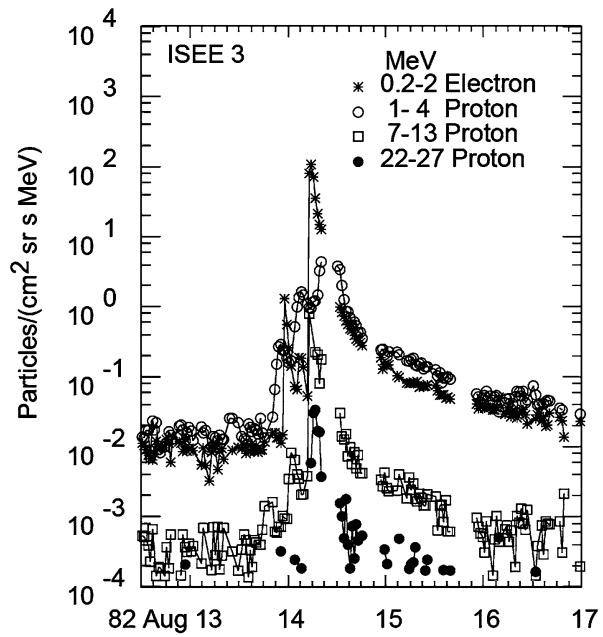

Interplanetary coronal mass ejections (ICMEs) produce SEPs that have highly variable intensity-time profiles that are different from the flare-related particle signatures. ICMEs may extend in solar longitude from $\sim 50^{\circ}$ (full-width) to $100^{\circ}$ (full-width) for more energetic events, their associated shock front can be even broader (Wimmer-Schweingruber et al., 2006; and references therein). Thus, as Figure 3 shows, the observed SEP intensity - time profiles will differ for the same CME event depending on where the observer is longitudinally connected to the CME shock, as discussed by Cane, Reames, and von Rosenvinge (1988), Reames (1999), and Cane and Lario (2006). It is the ICME shock that accelerates the particles magnetically connected with it, energizing the particles to intensity levels that can be greater than those related to CIR or impulsive flare events.

Solar events can occasionally occur during solar minimum and more frequently during the declining phase of the solar cycle. When solar events do occur during the minimum period, they can produce a SEP population that is mixed in with the CIR particle population such that the intensity - time profiles for this situation could be a combination of those that were shown in Figures 1 to 3 . During such mixed event periods, the use of particle composition information can help to distinguish the different sources of the energetic particles. For example, the ${ }^{3} \mathrm{He}$ isotope, which is rare in the solar wind, is a very good indicator for impulsive events since they are the only known source (Desai et al., 2006a; Mewaldt et al., 2007).

With the extended solar minimum period of SC 23 , there is an opportunity for us to examine the overall organization of the near-ecliptic energetic particles at $1 \mathrm{AU}$ for a much longer solar minimum period than is typical. From this study, we wish to understand how the CIR particle characteristics such as intensity and recurrence depend on the solar wind structure, especially the steepness and strength of their associated stream interactions, and whether the presence of SEPs affect the CIR events, such as increasing their intensities.

Our study is motivated by that of Sanderson et al. (1998) which used Wind spacecraft data (Ogilvie and Desch, 1997) to examine how the current sheet and compression regions controlled the observed CIR-related particle intensities at $1 \mathrm{AU}$ during the solar minimum period between Solar Cycles 22 (hereafter SC 22) and 23. Their main conclusions were that the tilt and warp of the heliospheric current sheet plays an important role in determining the shape and pattern of high-speed solar wind streams and compression regions, all of which influence the intensities and occurrences of the energetic particles. Simply stated, the CIR 


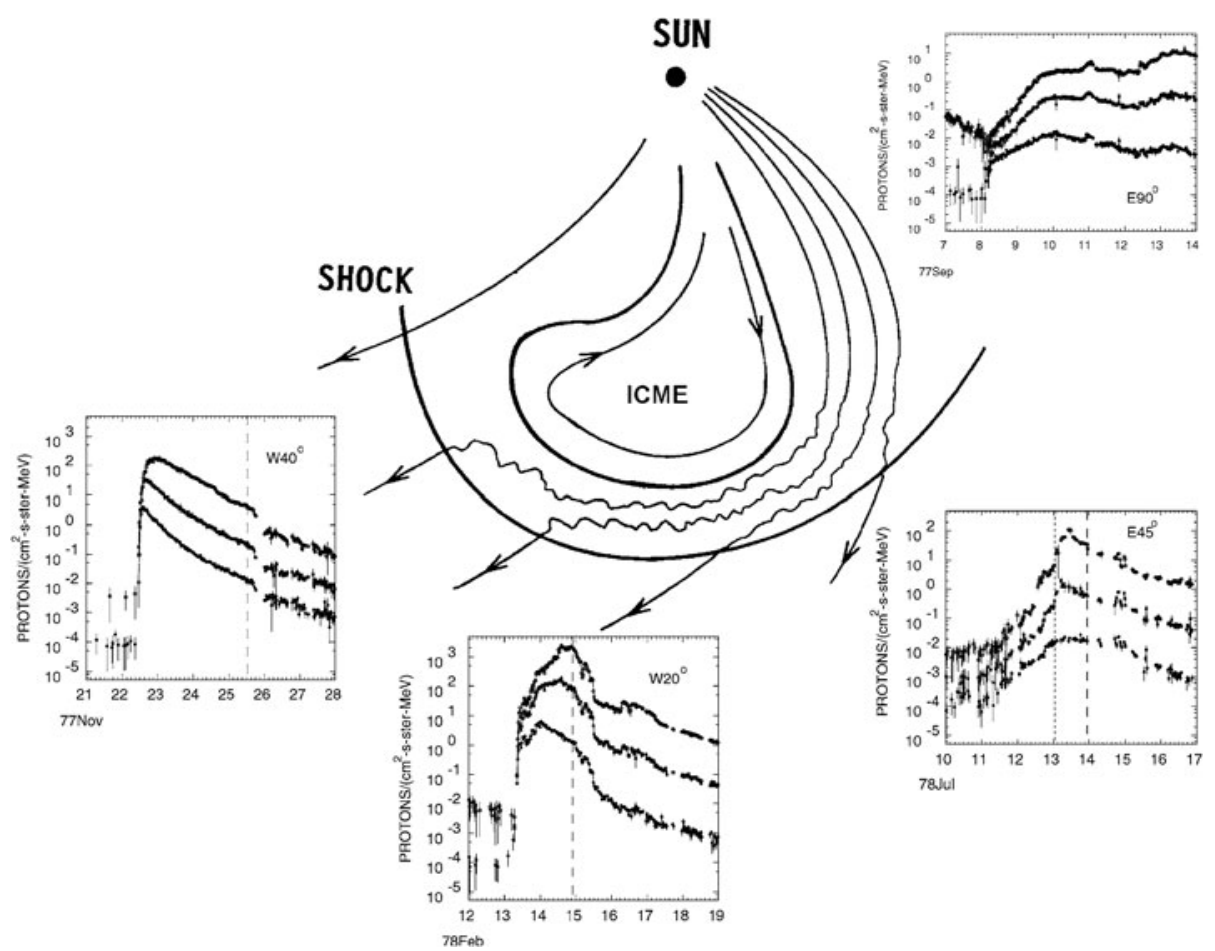

Figure 3 A cartoon illustration of a CME and its related shock. Also shown are time-intensity profiles for protons of $\sim 5, \sim 15$, and $\sim 30 \mathrm{MeV}$, as observed by the IMP-8 spacecraft. The profiles differ in shape for a given $\mathrm{CME}$ event depending on where the observer is located in solar longitude with respect to the CME ejecta and its related shock front. Dashed lines denote the times of the shock passages. Figure from Cane and Lario (2006).

particle events are organized by the structure of the solar wind streams. However, it is unclear whether variations in CIR particle event intensities are solely due to the characteristics of the solar wind stream interaction regions.

We will focus on the declining-to-minimum phase of SC 23, specifically, the $>6.5$ year period from 23 January 2003 to 13 October 2009 (Carrington rotations 1999 to 2088). We use particle data from the Electron, Proton, and Alpha Monitor (EPAM, Gold et al., 1998) and the Ultra-Low Energy Isotope Spectrometer (ULEIS, Mason et al., 1998) onboard the Advanced Composition Explorer (ACE) spacecraft located in orbit about the sunward Lagrangian point $\left(\mathrm{L}_{1}\right)$. We obtain the Level-2 hourly averaged EPAM data from the ACE Science Center (http://www.srl.caltech.edu/ACE/ASC/) and the ULEIS Level-2 hourly data from the NASA Goddard Space Flight Center Space Physics Data Facility (http://spdf.gsfc.nasa.gov/). For the plasma and magnetic field measurements we use the hourly OMNI data sets obtainable from http://omniweb.gsfc.nasa.gov/.

In Section 2 we discuss the global characteristics and recurrent features that are observed in the solar wind structure at $1 \mathrm{AU}$ for our period of interest. In Section 3 we investigate the organization of the energetic particles and how they relate to the solar wind structure. In Section 4 we evaluate the types of particle events that occurred and how they may be related to the particle intensities observed. In Section 5 we compare some of the results for this minimum period with those from SC 22. Finally in Section 6 we summarize our results. 

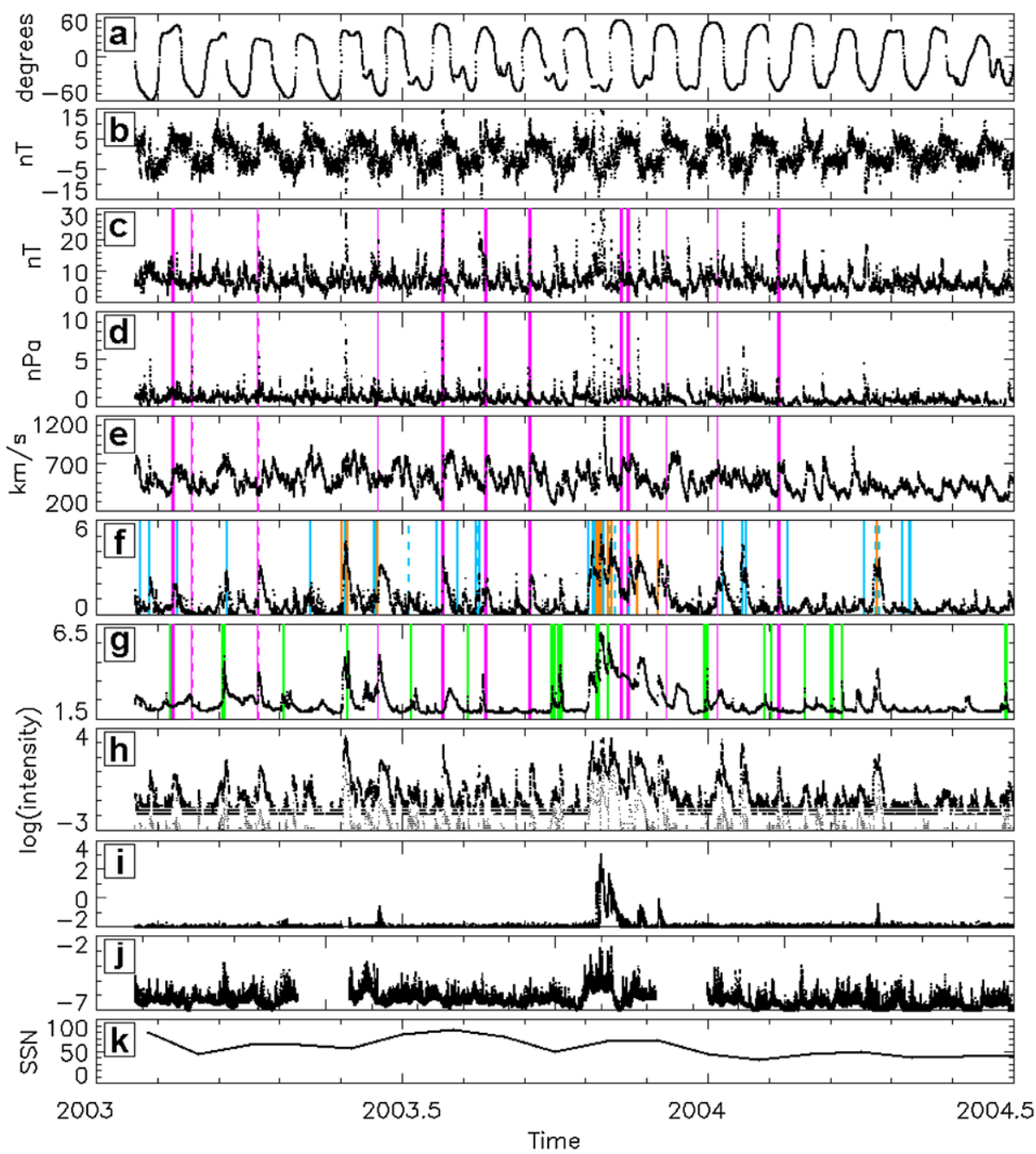

Figure 4 Time series from 2003 to 2004.5 of the (a) calculated distance of the HCS neutral line from the solar equator, (b) radial magnetic field, (c) total magnetic field, (d) dynamic pressure, (e) velocity, (f) EPAM 0.31 to $0.58 \mathrm{MeV}$ energetic ions, (g) EPAM 0.053 to $0.103 \mathrm{MeV}$ energetic electrons, (h) ULEIS 0.32 to $0.453 \mathrm{MeV} \mathrm{n}^{-1}{ }^{4} \mathrm{He}$ (thick black) and ULEIS 0.32 to $0.64 \mathrm{MeV} \mathrm{n}^{-1} \mathrm{Fe}$ (thin black) ions, (i) GOES-10 15 to $40 \mathrm{MeV}$ protons, (j) GOES-10 1-8 $\mathrm{X}$-ray flux data, and (k) monthly sunspot numbers. Color bars denote start times of various stream and solar events that are discussed in Section 4: thin magenta for SIR and thick magenta for CIR events, cyan for CME events, orange for SEP particle events, and green for beamed-electron events. The related shock event times are plotted as dashed bars using the same color scheme. We use hourly averaged data for (b) to (h) and 5-minute averaged data for (i) and (j).

\section{Solar Wind Structure}

Figures 4, 5, 6, and 7 summarize the primary data during our period of study in a standard time series format. These include the key parameters for a study of solar wind structure relationships to the energetic particle characteristics. The top panel (a) shows the approximate heliolatitude of the ecliptic plane from the heliospheric current sheet (HCS), calculated 

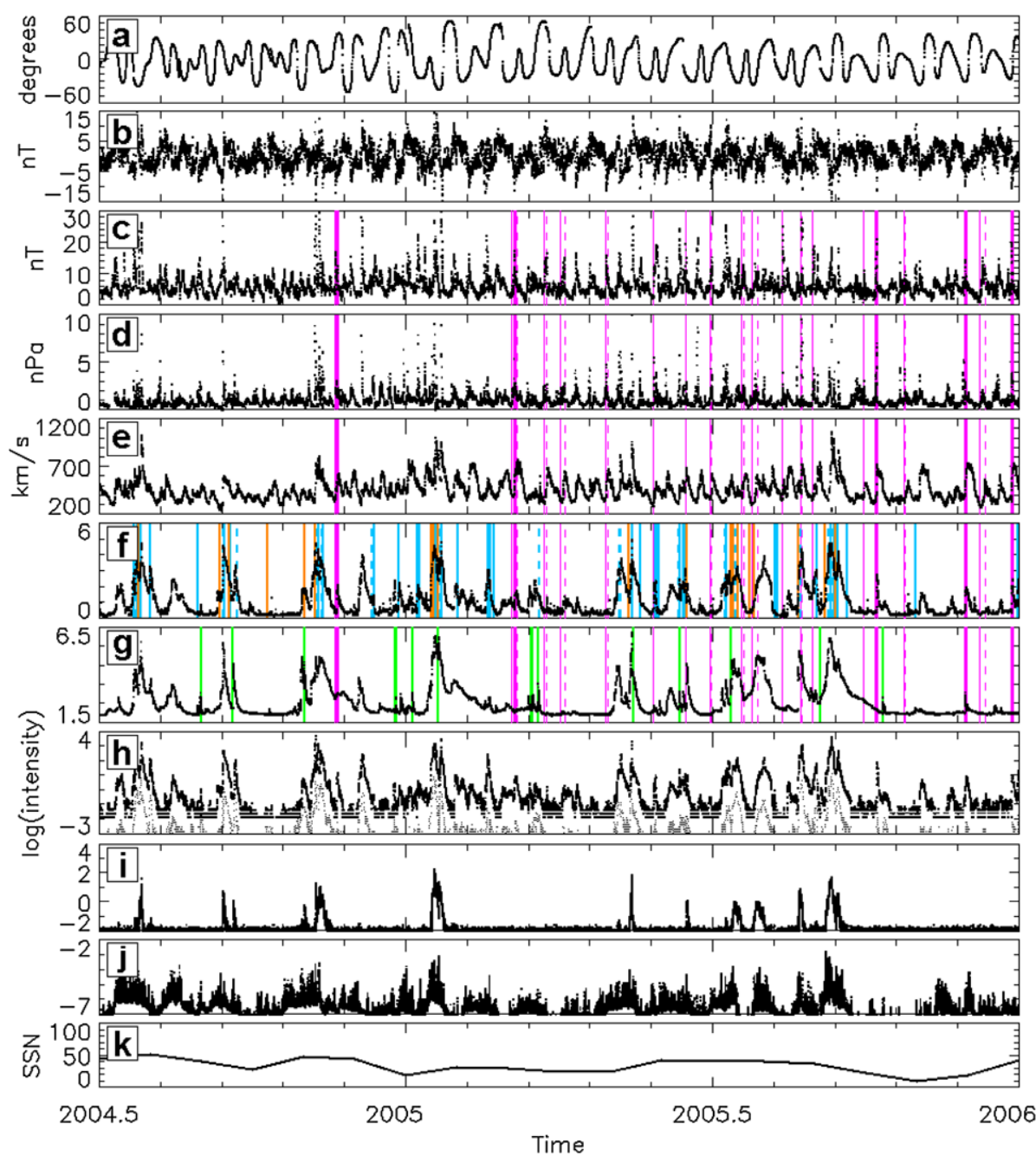

Figure 5 As for Figure 4 but from 2004.5 to 2006.

from the photospheric field synoptic map-based Potential Field Source Surface models of the coronal magnetic field (Schatten, Wilcox, and Ness, 1969; Altschuler and Newkirk, 1969). The progression from Figures 4 through 7 illustrates the simple dipolar (or two sector per solar rotation) structure of the HCS in the early part of the study interval, compared to the gentler, more quadrupolar warp observed in the later parts. The flattening of the warp during the last three years is notable and is expected during the descent into a solar minimum period. The OMNI radial and total magnetic fields (panels (b) and (c)) indicate the interplanetary field polarity and strength at $1 \mathrm{AU}$, respectively. These are followed by the solar wind dynamic pressures and velocities (panels (d) and (e)). The next four panels contain samples of energetic particle measurements from the ACE (panels (f) to (h)) and GOES (panel (i)) spacecraft, providing an overview of the ion and electron populations at energies $>100 \mathrm{keV}$. The last two panels ((j) and (k)) provide the GOES X-ray flux and the monthly sunspot numbers for context. 


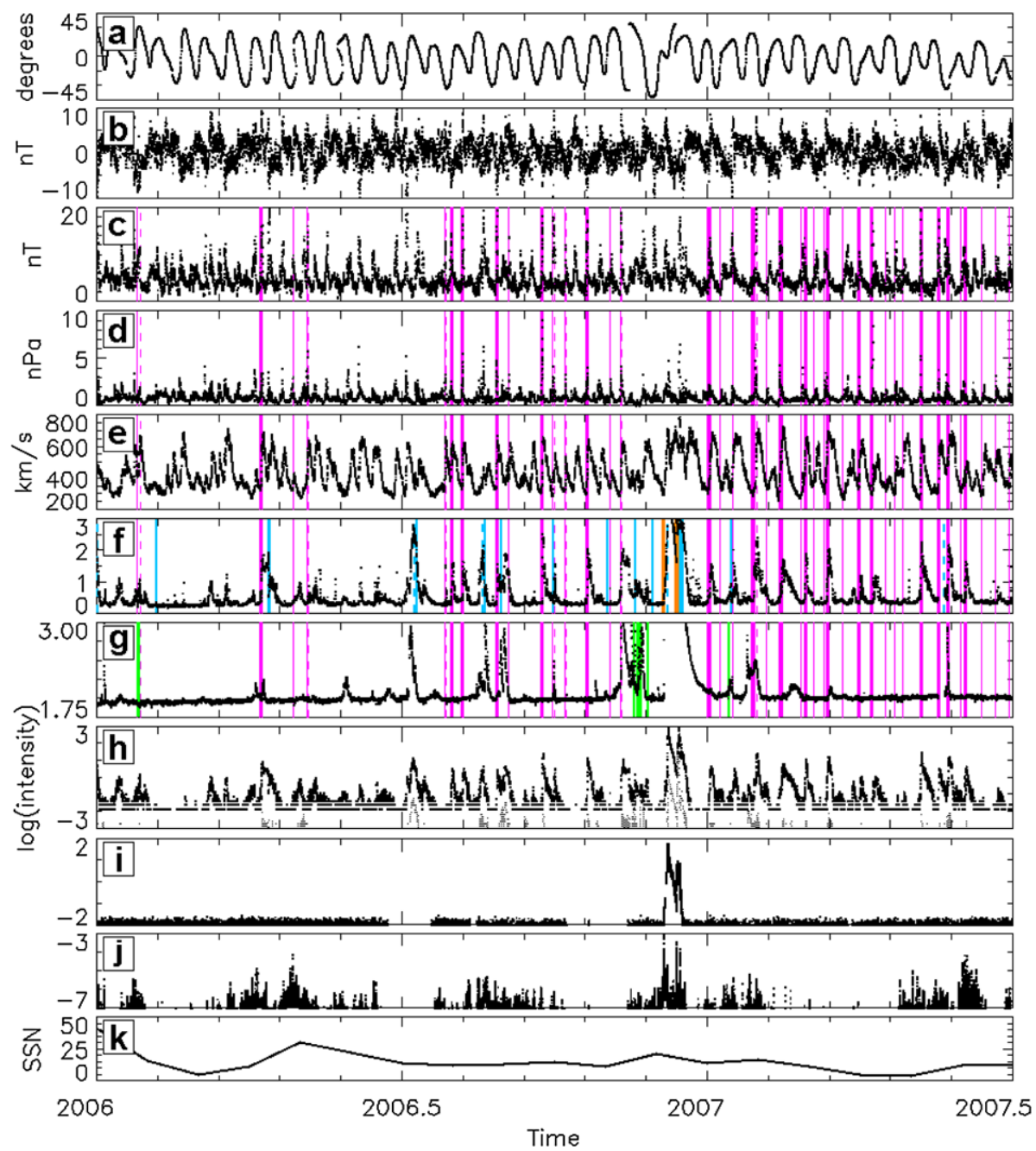

Figure 6 As for Figure 4 but from 2006 to 2007.5.

To get a better sense of the recurring features of the solar wind structure over a large range of solar rotations (e.g., one Carrington rotation, which takes 27.2753 days as viewed from Earth), we adopt a different plot style to display the parameters shown in Figures 4 to 7 . Figure 8 shows the color contour of the data, where the $x$-axis displays the Carrington rotation (CR) number (1999 to 2088) with the corresponding rotation commencement date indicated every 10 rotations, the $y$-axis displays the time in days since the beginning of the CR (1 to 27.3), and the color represents the value of the parameter. Similar types of plots were used in Sanderson et al. (1998) and also by Lee et al. (2009b) to analyze the solar wind and interplanetary field structure over the previous solar cycle. This format brings out recurrent versus nonrecurrent behavior and also allows relationships to be visualized. Both the time series and color contour plots will be used concurrently in the discussion below to describe the features of solar wind structure. 


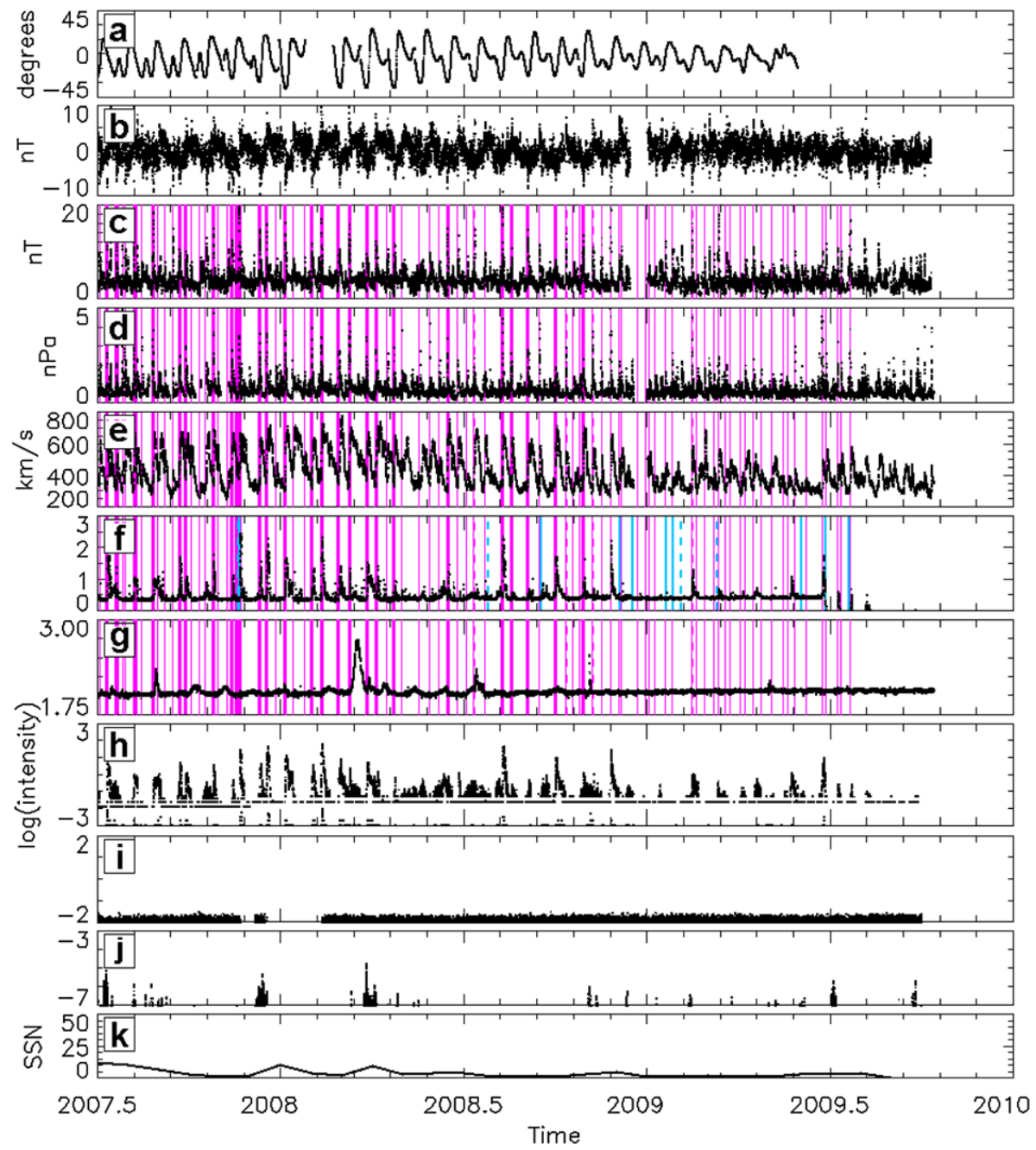

Figure 7 As for Figure 4 but from 2007.5 to 2010.

We begin by investigating the structure of the HCS at 1 AU. Figure 8(a) shows the color contour of the heliographic position of the solar neutral line at $1 \mathrm{AU}$ for our period of study. The distance of the neutral line from the solar heliographic equator was calculated for a radial distance of $2.5 R_{\odot}$ from the potential field source surface coefficients (C.N. Arge, private communication, 2009) derived from synoptic maps constructed from archived Mount Wilson Observatory magnetograph images. The results were linearly time-shifted by five days, the time for the solar wind to reach $1 \mathrm{AU}$ at an average speed of $\sim 345 \mathrm{~km} \mathrm{~s}^{-1}$, to allow cross-comparison with the solar wind and energetic particle observations at $1 \mathrm{AU}$. Note that we do not take into account the $\pm 7^{\circ}$ excursion of Earth in heliolatitude.

During our period of study, the HCS transitioned from a steep, two-sector structure (deep reds and blues in Figure 8(a)) to a flatter, four-sector structure (yellows and greens). From CRs 1999 to 2018 (i.e. declining phase), the HCS was substantially tilted and often inclined at $\geq 40^{\circ}$ (see Figure 4(a)). The HCS maintained its steepness when it transitioned to 

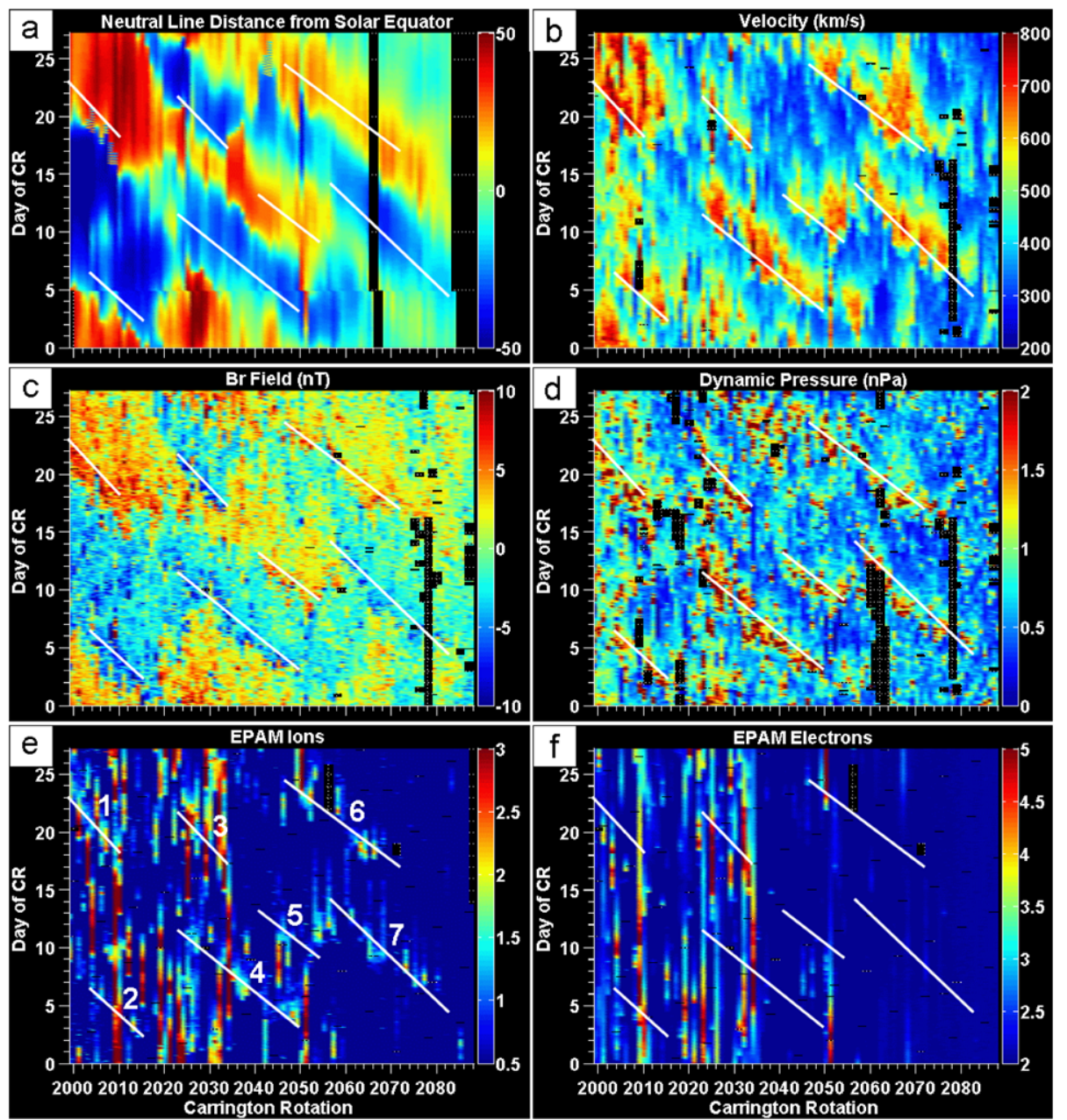

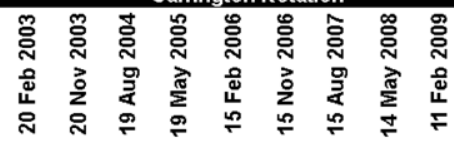

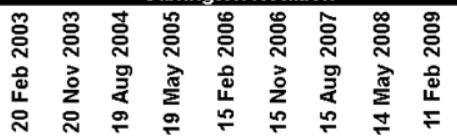

Figure 8 Shown are $1 \mathrm{AU}$ color contour plots from CRs 1999 to 2088 for (a) the calculated distance (degrees) of the HCS neutral line from the solar equator, (b) velocity $\left(\mathrm{km} \mathrm{s}^{-1}\right)$, (c) radial magnetic field (nT), (d) dynamic pressure (nPa), (e) EPAM 0.31 to $0.58 \mathrm{MeV}$ energetic ions, and (f) EPAM 0.053 to $0.103 \mathrm{MeV} \mathrm{n}^{-1}$ electrons. The white slanted lines and the number 1 to 7 mark the locations of features discussed in the text. The black gaps shown throughout are bad or missing data.

a four-sector structure around CR 2018 (around 2004.5 in Figure 4(a)). However, after the transition the inclination angles fluctuated for a number of solar rotations (e.g., from CRs 2018 to 2032) before returning to a relatively steady maximum inclination angle of around $\pm 30^{\circ}$ (see Figure 5(a)). The HCS gradually decreased in steepness during solar minimum, but it became very warped. Around CR 2056 until the end of the interval shown in Figure 8(a), one of the northern folds in the HCS flattened out (green) so that the HCS is north of the equator for about one quarter of a rotation, and near or south of the equator for about 
three quarters of a rotation. This warping, which gave the ecliptic field a notable unbalanced polarity distribution, can also be seen in the time series after 2007.3 (Figures 6(a) and 7(a)).

As expected, the pattern of the magnetic sectors is similar to that in the HCS (compare Figures 8(c) with 8(a)). During our study interval, the solar magnetic field was directed inward at the solar north pole and outward at the solar south pole. Consistent with this, the interplanetary magnetic field (IMF) at Earth is generally directed away from the Sun (radial field is positive) when the HCS is displaced to the north, and toward the Sun (radial field is negative) when the HCS is displaced to the south. The timing of the observed magnetic sector and the calculated HCS sector boundaries are also noticeably similar, particularly during the periods between CRs 1999 to 2018 as well as CR 2060 to the end of interval. Thus in general, our manual time-shifting by five days of the calculated HCS structure at $2.5 R_{\odot}$ to $1 \mathrm{AU}$ is a good approximation.

Throughout our study interval, regions of compressed fields can be seen at the leading edges of the magnetic field sectors (either in red for away or deep blue for toward the Sun, Figure 8(c)). From 2003 to 2006 during the declining phase, the time series (Figures 4(c) and 5(c)) show the peak total magnetic field strength often reached or exceeded $20 \mathrm{nT}$. Afterward, during the approach to the solar minimum period, the peak total field strength was mostly below $20 \mathrm{nT}$ (Figures 6(c) and 7(c)). We note that during the extended minimum period, the peak total field strengths were $<10 \mathrm{nT}$, whereas the radial field strengths were $\pm 5 \mathrm{nT}$ or less (see Figure 7(c), 2009.2 to 2009.5 and 2009.7 to 2009.8).

Field values of $\geq 30 \mathrm{nT}$ due to shocks and ICMEs following solar events were also observed during the declining phase. Panels (f) to (i) in Figures 4 to 7 show the $<1 \mathrm{MeV} \mathrm{n}^{-1}$ energetic ion and electron intensities as well as the $\geq 15 \mathrm{MeV} \mathrm{n}^{-1}$ protons. A number of major solar particle events can be identified in both the high and low energy data, the last series of events being in December 2006. Also, panel (j) in Figures 4 to 7 shows the $1-8 \AA$ $\mathrm{X}$-ray flux data, which identifies solar flare events. (We will discuss in greater detail the solar events and the energetic particles in the forthcoming sections.)

A striking feature is the many recurring fast solar wind streams shown in Figure 8(b). Using slanted white lines we manually mark the approximate locations of their leading edges, which roughly coincide with the stream interfaces as defined in the superposed epoch analysis by Gosling et al. (1978). Some recurrent streams spanned over 20 solar rotations, where one such sequence of streams occurred between Days 3 to 15 during CRs 2024 and 2050. This particular sequence originated from the northern solar hemisphere as indicated by the HCS and magnetic sector plots (Figures 8(a) and (c)), where the lines from Figure 8(b) are repeated to guide the eye. In general, the observed high-speed streams were located within the sector structure. The time series of the solar wind speed (Figures 4(e) to 6(e)) show that during the declining phase when the HCS was highly inclined, the velocity structure consisted of alternating periods of slow and fast solar wind, with maximum speeds ranging from $\sim 600 \mathrm{~km} \mathrm{~s}^{-1}$ to over $900 \mathrm{~km} \mathrm{~s}^{-1}$. The unsteadiness and fluctuations in the solar wind speeds are sometimes due to ICMEs, especially in the first third period of our study interval. During the minimum solar period that followed, the stream structure was generally weaker, such as during 2009 to 2009.8 in Figure 7(e) (or CRs 2080 to 2088 in Figure 8(c)), though the speeds still exceeded $\sim 500 \mathrm{~km} \mathrm{~s}^{-1}$ on occasion. We note that the lowest stream maximum speeds observed were $\sim 350 \mathrm{~km} \mathrm{~s}^{-1}$ (see Figure 7(e) during 2009.4 to 2009.5, or Figure 8(b) during CRs 2082 to 2086).

Figure 8(d) shows that our period of study is also dominated by the high pressure ridges (red) associated with CIRs at the leading edges of the recurring streams, as marked by the slanted white lines from Figure 8(b). It is known that the high-speed streams driving the CIRs typically occur following the magnetic sector boundaries (Crooker et al., 1999; 
Gosling et al., 1978). A comparison of Figure 8(d) with (c) does indeed show that the high pressure ridges occur behind these boundaries. Similar to the high-speed streams, the pressure ridges appeared much weaker during the solar minimum period (e.g., 2009.4 to 2009.5 in Figure 7(d), or CRs 2082 to 2086 in Figure 8(d)).

The notable slanted patterns in the color contour plots in Figure 8 indicate that the recurring features of the solar wind parameters appear earlier with each passing Carrington rotation. For the high-speed streams, this implies that the coronal hole sources producing them have a rotation period less than the 27.3-day Carrington rotation. Because the Sun rotates differentially, the location of the source region will determine the periodicity for the high-speed streams produced. It might be more appropriate to use the 27-day Bartels length, e.g., Sanderson et al. (1998). Over 20 rotations, the additional 0.3 day per rotation on a Carrington rotation scale amounts to 6 days, which is close to the slope of the recurrent features observed in Figure 8. However, to be consistent with prior studies (see, e.g., Lee et al., 2009a, 2009b), we use the 27.3-day Carrington rotation length, which represents a mid-latitude (or average) value and is readily associated with solar observations.

\section{Organization of Energetic Particles}

In Figure 8(e), the intensities of the 0.31 to $0.58 \mathrm{MeV}$ energetic ion population (mainly protons) as measured by the ACE EPAM instrument are shown. For this figure, the log of intensity is shown on the color scale. From the comparison with the slanted white lines, which as previously mentioned approximately indicate the leading edges of the solar wind streams, we identify the stream-associated recurrent particle enhancements indicated by the following groups of features: Days 18 to 23 of CRs 1999 to 2010 (hereafter called Feature 1), Days 2 to 7 of CRs 2004 to 2016 (hereafter called Feature 2), Days 17 to 22 of CRs 2022 to 2034 (hereafter called Feature 3), Days 3 to 12 of CRs 2022 to 2050 (hereafter called Feature 4), Days 10 to 13 of CRs 2040 to 2054 (hereafter called Feature 5), Days 17 to 25 of CRs 2040 to 2072 (hereafter called Feature 6), and Days 5 to 15 of CRs 2056 to 2084 (hereafter called Feature 7). Note that these recurring features together extend essentially throughout the whole period of our study, where Features 1 to 5 occur during the declining phase and Features 6 and 7 occur during the minimum period of the solar cycle. The time series of the same data set are also shown in panel (f) of Figures 4 to 7.

The recurrent ion particle enhancements (labeled 1 to 7 in Figure 8(e)) are co-located with the compression regions where the dynamic pressure values are high (Figure 8(d)) and located behind the magnetic sector boundaries (Figure 8(c)). When comparing the overall intensities of these recurrent particle enhancements, Feature 7 has the weakest intensities in comparison with Features 1 through 6. The weak signatures of the Feature 7 intensities can also be seen in Figure 6(f) and 7(f), after 2007.3. It is at this time that one of the northern folds of the four-sector HCS structure flattened out (Figure 8(a)). In addition, no significant recurrent ion particle enhancements were observed after CR 2080, when the recurrent solar wind streams were very weak (see Figure 8(b)).

During the declining phase, the association between the Features 1 to 5 recurrent ion intensities and the observed high pressure ridges is obscured by signatures related to solar activity. From 2003 to 2007 (panel (f) in Figures 4 to 6), there were periods of high intensity values that were well-above the background values. In Figure 8(e), these solar event-related intensities appear as vertical features, particularly during CRs 1999 to 2052. Similar vertical features can also be seen when we plot the synoptic views for the GOES-10 (Aschwanden, 1994) 15 to $40 \mathrm{MeV}$ energetic protons and 1-8 $\mathrm{X}$-ray data in Figure 9. Such vertical 


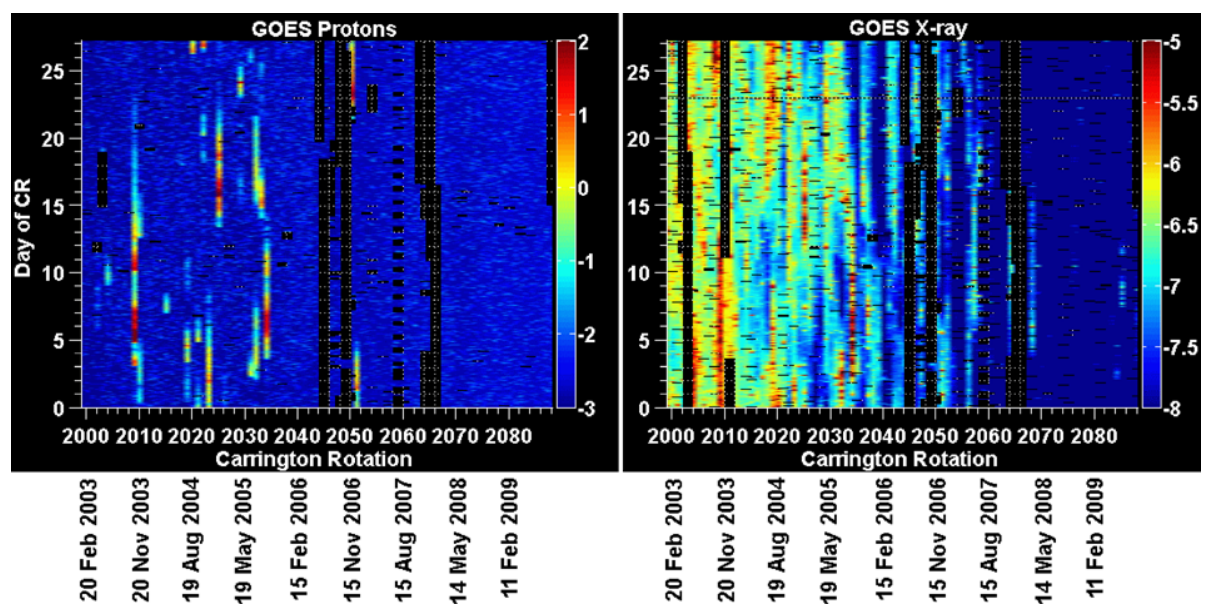

Figure 9 Color contour plots of the GOES-10 (left) 15 to $40 \mathrm{MeV}$ energetic protons and (right) $1-8 \AA$ $\mathrm{X}$-ray flux. We use the 5-minute resolution data set, where the black areas denote sections of bad data and the color scale is $\log$ of intensity.

features result from events that are extended in time but usually limited to one Carrington rotation (the exception being long-lived active regions that may produce multiple events during a sequence of rotations). From the energetic proton plot (left), the changeover from the SEP-dominated to the CIR-dominated epoch can be clearly seen as a transition from vertical to slanted patterns. In addition, the majority of the impulsive flare events ceased shortly after CR 2058 ( 2007.5 in Figure 7) as shown in the X-ray data (right), although some weaker events occasionally occurred during the minimum period.

Figure 8(f) shows the contour plot of intensities for the 0.053 to $0.103 \mathrm{MeV}$ energetic electrons, where periods of high intensities (vertical features) related to solar activity can be seen. It is noticeable that the electron enhancements due to the stream structures are not very prominent in these plots. Although we do see some weak enhancements near the slanted white lines that indicate the leading edges of the solar wind streams, these electron enhancements do not recur in a series like those for the energetic ions, especially after CR 2052 when the solar activity had subsided and the recurring streams were most prominent. This is further illustrated in Figures 6(g) and 7(g), where we plot the energetic electron time series for 2006 to the end of our study interval and overlay colored bars to mark various particle event periods (to be discussed in greater detail in the next section). Here, the thin magenta bars mark the stream interaction region (SIR) events (streams that do not occur for more than one solar rotation, as defined by Jian et al., 2006a) and the thick magenta bars mark the CIR events. From 2007 to 2008.5, for example, the recurrent ion enhancements (panel (f)) that are co-located with the magnetic compression regions and high-speed streams (panels (c) and (e), respectively), are not observed in general for the energetic electrons.

Since the first half of our study period was dominated by signatures due to solar events, we examine the intensities for specific ion species that are associated with such activity. It is known that Fe ions are good indicators of impulsive solar flare and ICME events, where impulsive flare events are Fe-rich and ICME events are Fe-poor (at energies of 0.5 to $10 \mathrm{MeV} \mathrm{n}^{-1}$ ) (Cane et al., 2006; Mewaldt et al., 2007). Panel (h) in Figures 4 to 7 shows the times series for the ULEIS 0.32 to $0.64 \mathrm{MeV} \mathrm{n}^{-1} \mathrm{Fe}$ ion intensities (thin black line). Although the overall intensity values are less than those for the energetic ions of similar 


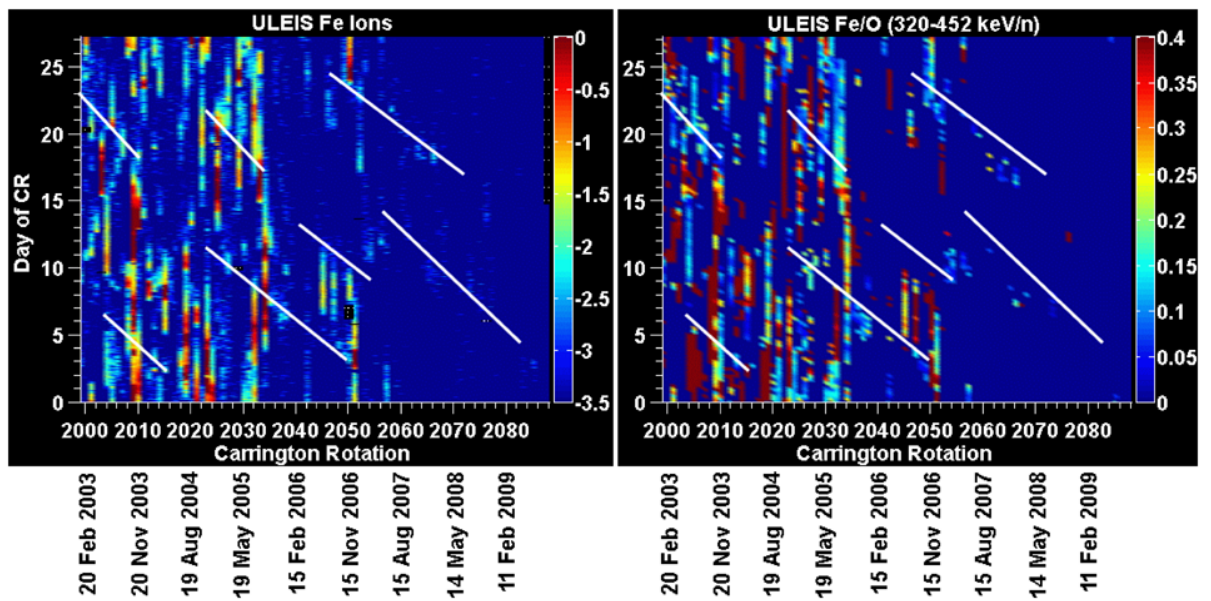

Figure 10 (Left) ULEIS 0.32 to $0.64 \mathrm{MeV} \mathrm{n}^{-1} \mathrm{Fe}$ ions and (right) ULEIS 6-hour resolution data for 0.32 to $0.452 \mathrm{MeV} \mathrm{n}^{-1} \mathrm{Fe} / \mathrm{O}$. The slanted white lines mark the locations of the leading edges of the recurring solar wind streams discussed in the text.

energies (compare with panel (f) in Figures 4 to 7), we do see periods of high intensities related to solar particle events. These periods also appear as vertical features in the Fe color contour plot (Figure 10, left), from CRs 1999 to 2052.

It should be noted that signatures of $\mathrm{Fe}$ can also be present during intense CIR events (see, e.g., Mason et al., 2008), and so the use of the Fe intensity is not necessarily a clean discriminator for solar activity-related material. The recurrent Fe ion intensities (e.g., Features 1 to 7) are observed (Figure 10, left), but their signatures are very weak in comparison with those for the EPAM ions (compare with Figure 8(e), or compare panels (f) and (h) in Figures 4 to 7). To further distinguish between CIR-related and SEP-related Fe signatures, we plot $\mathrm{Fe} / \mathrm{O}$ in Figure 10 (right) using the ULEIS 0.32 to $0.452 \mathrm{MeV} \mathrm{n}^{-1} \mathrm{Fe}$ and $\mathrm{O}$ 6-hour resolution data set. The relative abundance of Fe in CIR events is less than in SEP events, where the CIR average of $\mathrm{Fe} / \mathrm{O}$ is $\sim 0.088 \pm 0.007$ (Mason et al., 2008) and the SEP average is $\sim 0.404 \pm 0.047$ (Desai et al., 2006b). The CIR-related Fe signatures for values of $\mathrm{Fe} / \mathrm{O} \sim 0.1$ are shown in light blue. These CIR-related signatures are located throughout our period of study, including at the locations of the Features 1 to 7 intensities as marked by the slanted white lines. The SEP-related Fe signatures are shown in red for Fe/O 0.4. Figure 10 (right) clearly shows the decline of the Fe-enriched events as solar minimum was approached, after CR 2052. Many of the Fe/O values of $\sim 0.4$ coincide with the vertical patterns of high Fe intensities seen in Figure 10 (left).

The rare ${ }^{3} \mathrm{He}$ isotope is also a very good indicator of impulsive events since they are the only known source (Desai et al., 2006a; Mewaldt et al., 2007). In this study, however, we do not show the ${ }^{3} \mathrm{He}$ intensities since most of the data are mixed in with spill-over from ${ }^{4} \mathrm{He}$. Instead, we show the ULEIS 0.32 to $0.453 \mathrm{MeV} \mathrm{n}^{-1}{ }^{4} \mathrm{He}$ intensities (Figure 11, left) and the ${ }^{3} \mathrm{He} /{ }^{4} \mathrm{He}$ values calculated from the ULEIS 0.32 to $0.452 \mathrm{MeV} \mathrm{n}^{-1}{ }^{3} \mathrm{He}$ and ${ }^{4} \mathrm{He} 6$-hour resolution data set (Figure 11, right). For the ${ }^{3} \mathrm{He} /{ }^{4} \mathrm{He}$ values, we removed the ${ }^{4} \mathrm{He}$ spill-over in the ${ }^{3} \mathrm{He}$ data by omitting values of ${ }^{3} \mathrm{He} /{ }^{4} \mathrm{He}<5 \%$. From Figure 11 (left), the recurrent features are observed for ${ }^{4} \mathrm{He}$. Although the intensities are relatively weaker than the EPAM energetic ions, the details of the signatures are very similar to those shown for the EPAM ions (compare panel (h), thick black series, with (f) in Figures 4 to 7). The most prominent 

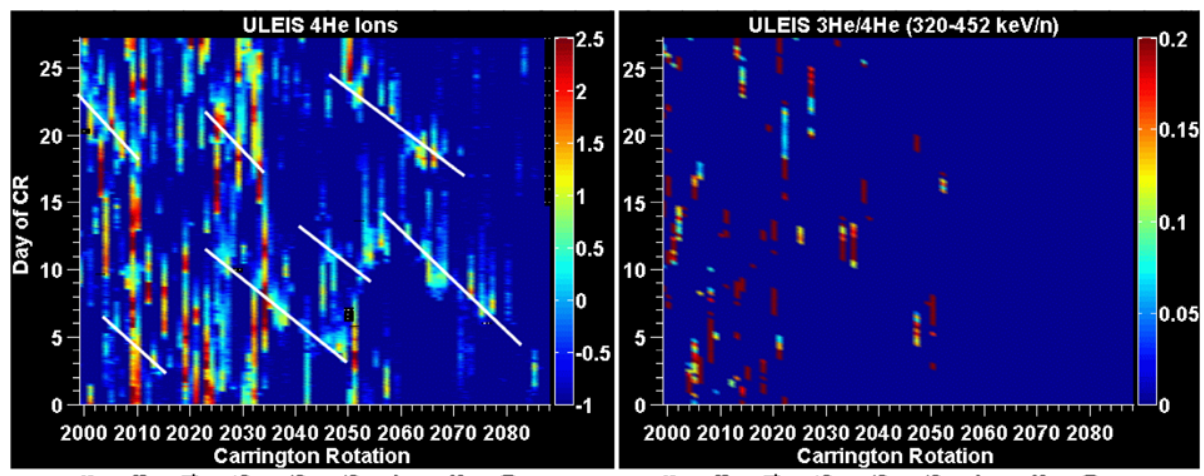

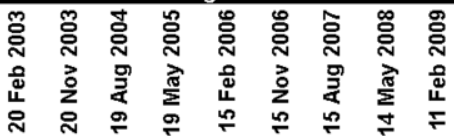

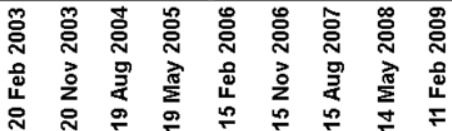

Figure 11 (Left) ULEIS 0.32 to $0.453 \mathrm{MeV}^{4} \mathrm{He}^{-1}$ ions and (right) ULEIS 6-hour resolution data for 0.32 to $0.452 \mathrm{MeV} \mathrm{n}^{-1}{ }^{3} \mathrm{He} /{ }^{4} \mathrm{He}$. The slanted white lines mark the locations of the leading edges of the recurring solar wind streams discussed in the text.

events in Figure 11 (right) are the ${ }^{3} \mathrm{He}$-rich events that occurred between major SEP events. The decline in the ${ }^{3} \mathrm{He}$-rich events as the solar cycle progressed toward the minimum phase can be clearly seen, although we note that ${ }^{3} \mathrm{He}$-rich events during quiet conditions have been reported by Mason et al. (2009b) but they do not appear in Figure 11 (right) because of low statistics.

\section{Particle Events}

In Figure 12 we further examine the various particle events that contribute to the observed energetic ion enhancements shown in Figure 8(e) and repeated in Figure 12(a). For each type of particle event, we overplot symbols in panels (b) though (e) that indicate the reported event onset times on top of the energetic ion color contour plot. We note that the juxtaposition of a particular intensity structure and a particle event does not necessarily mean that the structure is associated with the source of the event particles. Similarly, the detection of an energetic particle event without an apparent intensity feature association does not mean there is none, as a connection to the event may be remote, via magnetic field lines connecting to the observer. For example, a CIR could pass by at the time of a SEP event from the far west in solar longitude, which has no associated structures in near-Earth space at $1 \mathrm{AU}$. Nevertheless, such plots can call attention to the complications of particle-feature and event associations and also illustrate that they are often ambiguous.

In Figure 12(b) we overplot circles on top of the ion color contour plot to identify events due to SIR events, and asterisks to mark the times of the related SIR shock events. We use the 'SIR Start UT' and 'Shock UT' event times based on ACE and Wind spacecraft observations from published lists by Jian et al. (2006a, 2008). The listed events are a mixture of both SIR and CIR events; if an SIR recurred on two or more solar rotations, it was also considered a CIR. The event list extends to late-July 2009 (CR 2085). A majority of SIRs and their related shocks occur at or near the Features 4 to 7 recurrent ion intensities that are correlated with the high dynamic pressure ridges. There is a noticeable event gap between CRs 2010 and 


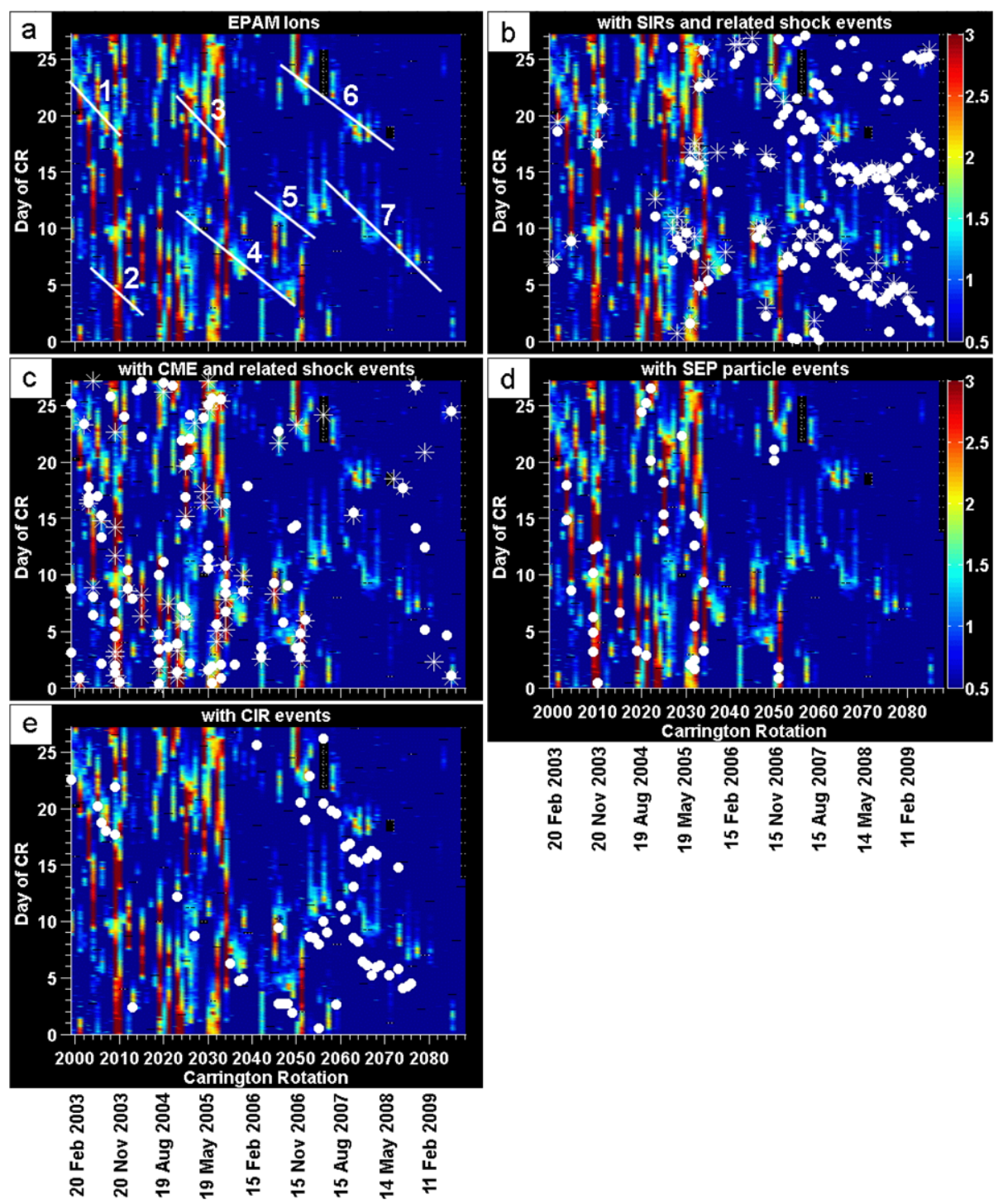

Figure 12 ACE EPAM 0.31 to $0.58 \mathrm{MeV}$ ion intensities overplotted with (b) SIR events (circles) and their related shocks (asterisks), (c) CME events (circles) and their related shocks (asterisks), (d) SEP particle events, and (e) CIR-related particle events. The slanted white lines mark the locations of the leading edges of the recurring solar wind streams discussed in the text.

2020, probably when the HCS was slowly transitioning from a two- to four-sector structure. It is at this time, also, that there is a lack of strong and continuous high-speed solar wind streams (see Figure 8(b)). We also note the SIR events occurring after CR 2080 do not have noticeable corresponding particle enhancements in Feature 7.

In Figure 12(c) we plot near-Earth ICMEs and their related shocks, shown as circles and asterisks, respectively. The observed particle intensities that occurred around the same time are not necessarily caused by them, but are often related. The event times for the ICMEs are 

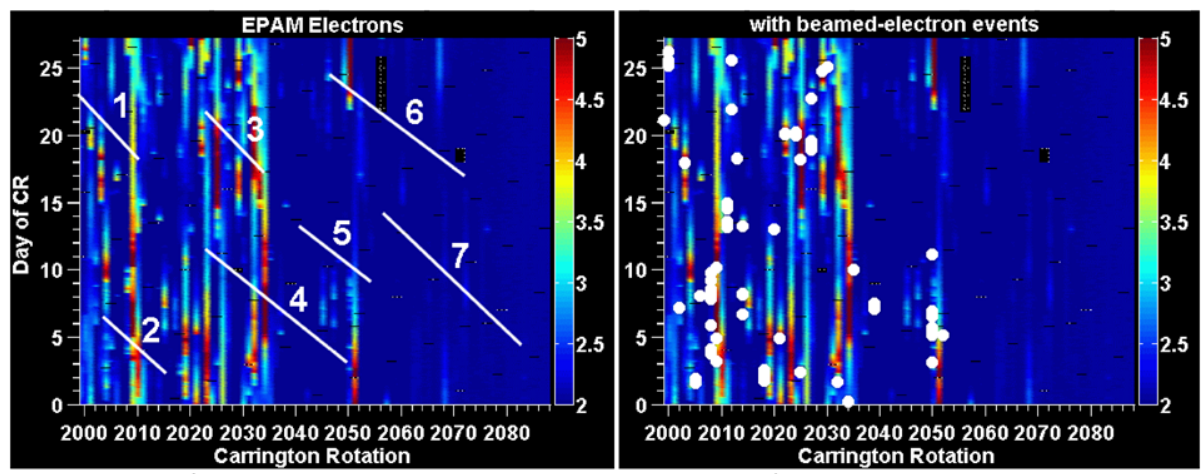

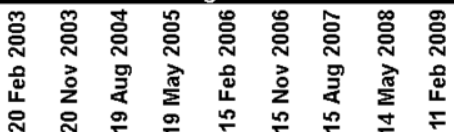

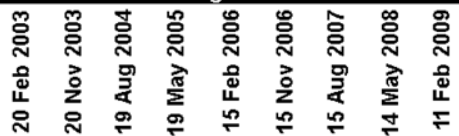

Figure 13 (Left) ACE EPAM 0.053 to $0.103 \mathrm{MeV}$ electron intensities overplotted with (right) beamed-electron events. The slanted white lines mark the locations of the leading edges of the recurring solar wind streams discussed in the text.

based on the plasma and magnetic field observations by ACE and Wind, and are obtained from a list compiled by I.G. Richardson and H.V. Cane that is available on the internet (http://www.ssg.sr.unh.edu/mag/ace/ACElists/ICMEtable.html; see also Cane and Richardson, 2003). We use the 'ICME plasma/field' start times from this list, which ends around late-July 2009 (CR 2085). For the ICME-related shocks observed by ACE and Wind, we use the shock start times from published event lists by Jian et al. (2006b, 2008). The list of shock events is also provided up to late-July 2009 (CR 2085). Figure 12(c) shows that the ICME events and their related shocks are concentrated roughly around CRs 1999 to 2052, during the active solar period, with a few sporadic events afterward. The ICME and related shock events correlate most with the vertical patterns of high ion intensities and not the Features 1 to 5 recurrent intensities that are related to the solar wind dynamic pressure, as expected. Because we only use the start times of the ICME events provided in the event lists mentioned, information regarding the durations of enhanced particle activity is not included.

Figure 12(d) shows SEP events that are either accelerated at the associated flare site or by the passage of a CME-driven shock obtained from a published list by Cane et al. (2006) for $>25 \mathrm{MeV}$ proton events extending through the end of year 2005 (CR 2038). Four additional events for year 2006 are included as well. The SEP event start times are based on the GOES soft X-ray maximum intensity or the start of the type III bursts if the flare was behind the solar limb. The events shown occur mostly during the declining phase of our study and overlap mainly with the vertical pattern of high ion intensities.

Figure 12(e) shows energetic particle (heavy ion) events related to CIRs. The start times are based on the event lists provided by Mason et al. (2008, 2009a), where the events are observed by ULEIS through the end of 2008 October (middle of CR 2076). As expected, the CIR events plot along the contours of high ion intensities that are correlated with the high dynamic pressure ridges (e.g., Features 1 to 7 intensities). There is also a noticeable event gap between CRs 2010 and 2020, as shown earlier for the SIR events in Figure 12(b).

In Figure 13 we examine events for the energetic electron particles. We repeat the contour plot of the energetic electrons (Figure 8(f)) with the Features 1 to 7 labeled on the left panel, and in the right panel we overplot the impulsive beamlike near-relativistic electron events 
(circles) observed by ACE EPAM near 1 AU. These events are associated with solar flare events observed between $\mathrm{W} 30^{\circ}$ and $\mathrm{W} 80^{\circ}$, where $\mathrm{W} 60^{\circ}$ is the ideal average magnetic connection longitude to Earth. The list, extending to the beginning of year 2007 (CR 2052), is a continuation of the original list published by Haggerty and Roelof (2002) and can be found at http://www.srl.caltech.edu/ACE/ASC/DATA/level3/index.html. Although it was reported by Haggerty and Roelof (2002) that these particular electrons are accelerated by CME-driven shocks, their recent study of the entire SC 23 period indicate that the electrons are a mixture of shock- and flare-accelerated electrons (Haggerty and Roelof, 2009). Figure 13 (right) shows that the electron events overlap with many of the vertical features (high intensities) due to solar activity, where the frequency of the reported electron events declined as expected as solar minimum was approached.

\subsection{Mixed Event Periods}

During the declining phase, the CIR particle population is mixed in with the population of energetic particles that are related to solar activity. We thus take a closer look at the energetic ion and electron particle enhancements that occurred at this time and revisit Figures 4 and 5. These figures are divided into two time intervals: 2003 to 2004.5 and 2004.5 to 2006, respectively. The solid color bars are plotted to represent the following particle events: thin magenta for SIR and thick magenta for CIR events, cyan for CME events, orange for SEP particle events, and green for beamed-electron events. The related shock event times are plotted as dashed bars using the same color scheme.

Panels (f) and (g) in Figures 4 and 5 show on several occasions the energetic particle enhancements that occurred during or around the times of both stream and solar-related event periods. One such example is the occurrences of two near-Earth ICME events on 15 and 16 June 2003 and one SIR event on 18 June 2003 (event times based on an online list by I.G. Richardson and H.V. Cane and a published list by Jian et al., 2006a, respectively). Shortly after these events, comparable enhancements in the energetic ion (panel (f)) and electron (panel (g)) intensities were observed. In another example, shortly after 2004.0, one stream event occurred (6 January 2004) followed by a near-Earth ICME event (9 January 2004). The related energetic ion and electron enhancements can be seen, though the electron enhancement is relatively weak. In this particular example, the rise of the ion intensity commenced with the passage of the stream followed by a strong and relatively sharp peak that occurred with the passage of the ICME disturbance. Other mixed event periods were also observed throughout 2005, especially the period between 2005.4 and 2005.7. During the minimum period, particularly after 2007 (Figures 6 and 7), the stream events dominated with a few solar events occurring sporadically (see, e.g., Bucik et al., 2009). It is not clear from these new displays (e.g., Figure 12) that the intensities of the ICME shock-related events are affected by the presence of pre-existing SIR/CIR or ICME particle populations. The issue of seed populations is thus not further resolved by this study.

\section{Comparison with the Solar Cycle 22 Minimum Period}

To compare some of our results with those reported by Sanderson et al. (1998), we focus on the portion of the solar cycle where the sunspot numbers (SSN) are comparable. The period studied by Sanderson et al. (1998) began around November 1994 (approach of Solar Cycle 22 to 23 minimum period) and had SSN $\sim 45$. Hence, in this section we narrow our study interval and begin our comparison on January 2006 (between CRs 2038 and 2039 in 
Figure 14 (a) Monthly sunspot number (SSN) for the periods of interest. The light gray shaded area indicates the period of study by Sanderson et al. (1998), beginning around November 1994. The dark gray shaded area indicates the period of this study. (b) Zoomed-in plot indicating comparable minimum period of this study with that of Sanderson et al. (1998).

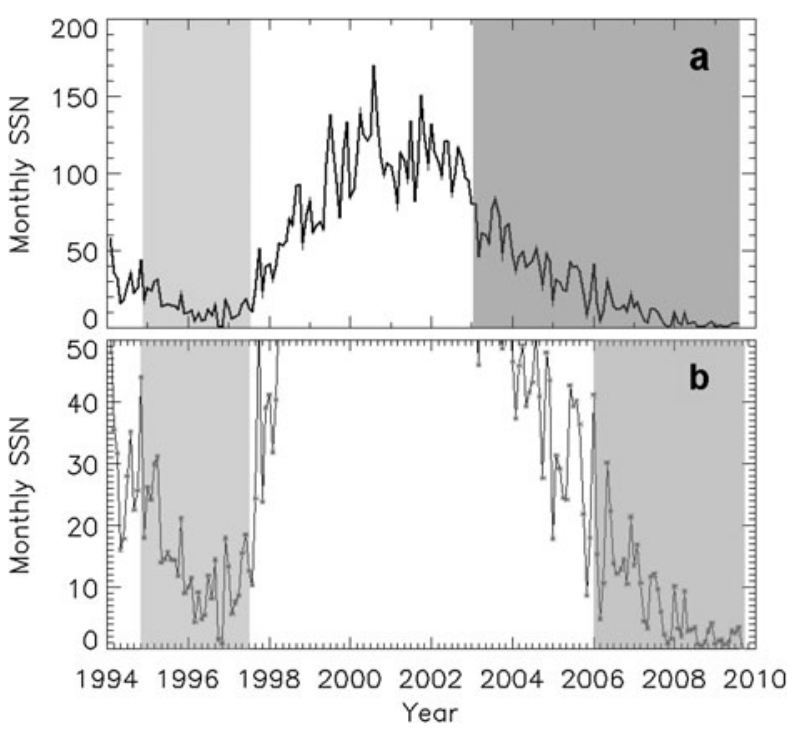

Figure 8) when the SSN was comparable (Figure 14(b)). It is clear from Figure 14 that the period with SSN $\leq 45$ following SC 23 is already longer than during the previous minimum period even though the rising phase of SC 24 has yet to commence by the end of our study period. To compare our results with those from the previous minimum period, we refer to Plates 1 to 3 in Sanderson et al. (1998).

\subsection{Solar Wind Structure}

For both minimum periods, the sector pattern, which followed closely the shape of the neutral line, had already transitioned from a steeper, two-sector structure to a flatter, four-sector structure. The HCS has maintained a four-sector pattern for the entire SC 23 minimum period thus far, although during the bottom of the minimum (2008 to late 2009, CRs 2064 to 2084) some warping occurred such that the HCS was north about one quarter of a solar rotation and near or south of the equator for about three quarters of a solar rotation. A similar warping also occurred during the bottom of the SC 22 minimum period but for a much shorter time interval (January to June 1996, CRs 1905 to 1909; see panels (c) and (d) of Plate 1 in Sanderson et al., 1998). Moreover, when we compare inclination angles, the HCS had a maximum tilt of $\sim 45^{\circ}$ during the SC 23 minimum period (see Figures 6(a) and 7(a)) in comparison to a maximum tilt of $\sim 30^{\circ}$ during the SC 22 minimum period (see panel (c) of Plate 1 in Sanderson et al., 1998).

The pattern of the high-speed streams also followed the pattern of the sectors, as expected, during both minimum periods. For the SC 23 minimum, there were several highspeed solar wind streams that were very long-lived (e.g. the streams that occurred during CRs 2022 to 2050 at Days 3 to 12 and also CRs 2056 to 2084 at Days 5 to 15 in Figure 8(b)). These recurring streams spanned over many solar rotations, up to $\sim 30$ at times, whereas during SC 22 the recurring streams spanning $\sim 10$ solar rotations were not quite as long-lived (see panel (d) of Plate 3 in Sanderson et al., 1998). We note that the slopes of the recurrent features seen throughout this study (e.g., Figure 8) are not observed in Plate 3 of Sanderson et al. (1998) since they use the 27-day Bartel rotation scale instead of the 27.3-day Carrington rotation scale. 
Typically during the minimum phase of the solar cycle, the polar coronal holes and their extensions would supply the high-speed streams observed in the ecliptic (see Luhmann et al., 2002 for a discussion). However, in a study by Lee et al. (2009b) where they were investigating the sources of the weaker ecliptic IMF, they found that for SC 23 there were many prominent low-to-mid latitude coronal holes that were supplying the high-speed streams observed at $1 \mathrm{AU}$. In contrast, the polar coronal holes and their extensions mostly supplied the high-speed streams during the SC 22 period. Luhmann et al. (2009) proposed that the weaker solar fields that occurred during the SC 23 minimum period led to more prominent low latitude coronal holes and therefore stronger high-speed streams.

A comparison was made in Lee et al. (2009b) for the distribution of the solar wind speeds and magnetic field strengths observed during the SC 22 and 23 minimum periods. Overall, the speed distribution for both minimum periods was comparable. In addition, the highspeed streams were reported to be well-formed for both periods, though for the SC 22 minimum there were fewer high-speed streams observed. For the interplanetary field strength, the values were reported to be $\sim 30 \%$ lower for the cycle 23 minimum period (McComas et al., 2008), which is also consistent with the Ulysses off-ecliptic observations reported by Smith and Balogh (2008).

\subsection{Energetic Particle Enhancements}

The energetic ion enhancements observed by ACE during the recent solar minimum period followed the pattern of both dynamic pressure and magnetic field enhancements associated with the stream interaction regions (e.g., Features 4 to 7 in Figure 8(e)). This was also the case for enhancements of similar energies observed by Wind during the previous solar minimum (see panel (e) of Plate 3 in Sanderson et al., 1998). For both cases, the recurrence of the ion enhancements occurred on the time scales of the recurring high-speed streams observed during the respective minimum periods. The electron enhancements associated with the compression regions were also observed during both minimum periods (similar energy ranges), although they did not always occur when the ion enhancements were observed nor did they recur with the high-speed streams (see Figures 4 and 7). When the electron intensities were observed, they were often weaker than the ion enhancements (for SC 22 compare red time series in panels (a) and (b) of Plate 2 in Sanderson et al., 1998; for SC 23 compare panels (f) and (g) in Figures 6 and 7 of this study).

Solar activity occurred sporadically for both solar minimum periods and produced particle enhancements for both the energetic ions and electrons. At times, these solar events commenced at or around the times of the streaming events, thus producing mixed signature profiles in the particle intensities. Both solar minimum periods have similar energetic particle event characteristics, with the differences only determined by the different solar wind structures and ICME event rates.

\section{Conclusions}

The unusually long solar minimum period of SC 23 has provided an opportunity to examine the large-scale organization of the energetic particles in the context of the ambient solar wind structure at $1 \mathrm{AU}$. We made an analysis of the SC 23 declining to minimum period, similar to the study by Sanderson et al. (1998) who conducted a global study using Wind observations of the association of energetic particles with CIRs for the minimum period of Solar Cycle 22. Their observations showed that the proton intensities followed a pattern of the solar wind compression regions, suggesting that the particle events are organized 
by the corotating structure of the solar wind streams. For our study, we utilized the in-situ OMNI solar wind plasma and magnetic field as well as the ACE particle data sets, spanning $>6.5$ years of observations from CRs 1999 to 2088 (January 2003 to October 2009) that includes the declining phase and the minimum period.

During the declining phase, we find that the HCS was inclined and warped, forming a two-sector interplanetary magnetic field structure at Earth. During the approach to solar minimum, the HCS maintained a relatively steep inclination and the IMF transitioned to a four-sector pattern. The HCS became less steep during solar minimum, but some warps were observed.

Also, throughout our period of study there were several steady, high-speed solar wind streams and corresponding high dynamic pressure ridges. Some of these streams recurred for many solar rotations, at times up to 30 rotations, indicating the coronal sources producing these streams were quite long-lived. In particular, these long-lived streams occurred during the period when the HCS was a four-sector structure. As solar minimum progressed, the high-speed streams and corresponding high pressure ridges became much weaker.

We find that the recurring energetic particle enhancements were associated with the longlived high solar wind pressure regions. The CIR-related particles were observed throughout our period of study, but during the declining phase there were also many solar events that occurred. Using various solar event lists that have been published or made available online, we plotted the start times of these events on top of the particle intensity plots in an attempt to distinguish some of the sources that may have produced the various particle signatures. We find that some of the observed particle events during the present solar minimum may be related to ICME or flare events (or both). As the decline in solar activity progressed, the observed particle signatures due to CIR events became dominant. However, very few significant recurrent energetic particle enhancements were observed between CRs 2080 and 2088, which is consistent with the overall weak high-speed streams observed during this time and also with the presence of several still-active regions from cycle 23. As we approach Solar Cycle 24, we expect to see an ongoing presence of CIR-related energetic particle intensities and a more frequent occurrence of the SEP event-related enhancements. The details of the evolution of energetic particle event types should depend on both the solar wind structure and active region behavior during the new rising phase, along the lines of the overall picture described in this study.

Acknowledgements The authors would like to thank C.N. Arge for providing the PFSS coefficients that enabled us to computing the HCS neutral line distance. The authors would also like to thank the NASA Goddard Space Flight Center Space Physics Data Facility (SPDF) and the ACE Science Center for providing access to their data sets.

Christina O. Lee thanks the editor and referee for their assistance in evaluating this paper.

This research was supported by the 2005-2006 National Defense Science and Engineering Graduate (NDSEG) Fellowship awarded by the Department of Defense (DoD) and the CISM project, which is funded by the STC Program of the National Science Foundation under agreement number ATM-0120950.

Open Access This article is distributed under the terms of the Creative Commons Attribution Noncommercial License which permits any noncommercial use, distribution, and reproduction in any medium, provided the original author(s) and source are credited.

\section{References}

Altschuler, M.D., Newkirk, G.: 1969, Magnetic fields and the structure of the solar corona. Solar Phys. 9, $131-149$. 
Aschwanden, M.J.: 1994, Irradiance observations of the $1-8$ Å solar soft X-ray flux from GOES. Solar Phys. 152, $53-59$.

Barnes, C.W., Simpson, J.A.: 1976, Evidence for interplanetary acceleration of nucleons in corotating interaction regions. Astrophys. J. 210, L91 - L96.

Bucik, R., Mall, U., Gomez-Herrero, R., Korth, A., Mason, G.M.: 2009, STEREO observations of energetic ions in corotating interaction regions during the May 2007 solar events. Solar Phys. 259, 361 - 380.

Cane, H.V., Lario, D.: 2006, An introduction to CMEs and energetic particles. Space Sci. Rev. 123, $45-56$.

Cane, H.V., Richardson, I.G.: 2003, Interplanetary coronal mass ejections in the near-Earth solar wind during 1996-2002. J. Geophys. Res. 108, 1156. doi:10.1029/2002JA009817.

Cane, H.V., Reames, D.V., von Rosenvinge, T.T.: 1988, The role of interplanetary shocks in the longitude distribution of solar energetic particles. J. Geophys. Res. 93, 9555-9567.

Cane, H.V., Mewaldt, R.A., Cohen, C.M.S., von Rosenvinge, T.T.: 2006, Role of flares and shocks in determining solar energetic particle abundances. J. Geophys. Res. 111. doi:10.1029/2005JA011071.

Crooker, N.U., Gosling, J.T., Bothmer, V., Forsyth, R.J., Gazis, P.R., Hewish, A., Horbury, T.S., Intriligator, D.S., Jokipii, J.R., Kota, J., et al.: 1999, CIR morphology, turbulence, discontinuities, and energetic particles. Space Sci. Rev. 89, 179-220.

Desai, M.I., Mason, G.M., Mazur, J.E., Dwyer, J.R.: 2006a, Solar cycle variations in the composition of the suprathermal heavy-ion population near 1 AU. Astrophys. J. 645, L81 - L84.

Desai, M.I., Mason, G.M., Gold, R.E., Krimigis, S.M., Cohen, C.M.S., Mewaldt, R.A., Mazur, J.E., Dwyer, J.R.: 2006b, Heavy-ion elemental abundances in large solar energetic particle events and their implications for the seed population. Astrophys. J. 649, 470-489.

Forsyth, R.J., Marsch, E.: 1999, Solar origin and interplanetary evolution of stream interfaces. Space Sci. Rev. 89, $7-20$.

Gold, R.E., Krimigis, S.M., Hawkins, S.E. III, Haggerty, D.K., Lohr, D.A., Fiore, E., Armstrong, T.P., Holland, G., Lanzerotti, L.J.: 1998, Electron, Proton, and Alpha Monitor on the Advanced Composition Explorer spacecraft. Space Sci. Rev. 86, $541-562$.

Gosling, J.T., Pizzo, V.J.: 1999, Formation and evolution of corotating interaction regions and their three dimensional structure. Space Sci. Rev. 89, $21-52$.

Gosling, J.T., Asbridge, J.R., Bame, S.J., Feldman, W.C.: 1978, Solar wind stream interfaces. J. Geophys. Res. 83, $1401-1412$.

Haggerty, D.K., Roelof, E.C.: 2002, Impulsive near-relativistic solar electron events: Delayed injection with respect to solar electromagnetic emission. Astrophys. J. 579, 841-853.

Haggerty, D.K., Roelof, E.C.: 2009, Probing SEP acceleration processes with near-relativistic electrons. In: AIP Conf. Proc. 1183, 3-10.

Jian, L.: 2008, Radial variation of large-scale solar wind structures. Ph.D. dissertation, Univ. California, Los Angeles.

Jian, L., Russell, C.T., Luhmann, J.G., Skoug, R.M.: 2006a, Properties of stream interactions at one AU during 1995-2004. Solar Phys. 239, 337 - 392.

Jian, L., Russell, C.T., Luhmann, J.G., Skoug, R.M.: 2006b, Properties of interplanetary coronal mass ejections at one AU during 1995-2004. Solar Phys. 239, 393-436.

Lee, C.O., Luhmann, J.G., Odstrcil, D., MacNeice, P.J., de Pater, I., Riley, P., Arge, C.N.: 2009a, The solar wind at $1 \mathrm{AU}$ during the declining phase of Solar Cycle 23: Comparison of 3D numerical model results with observations. Solar Phys. 254, $155-183$.

Lee, C.O., Luhmann, J.G., Zhao, X.P., Liu, Y., Riley, P., Arge, C.N., Russell, C.T., de Pater, I.: 2009b, Effects of the weak polar fields of Solar Cycle 23: Investigation using OMNI for the STEREO mission period. Solar Phys. 256, $345-363$.

Luhmann, J.G., Li, Y., Arge, C.N., Gazis, P.R., Ulrich, R.: 2002, Solar cycle changes in coronal holes and space weather cycles. J. Geophys. Res. 107, 1154. doi:10.1029/2001JA007550.

Luhmann, J.G., Lee, C.O., Li, Y., Arge, C.N., Galvin, A.B., Simunac, K., Russell, C.T., Howard, R.A., Petrie, G.: 2009, Solar wind sources in the late declining phase of Cycle 23: Effects of the weak solar polar field on high speed streams. Solar Phys. 256, $285-305$.

Mason, G.M., Sanderson, T.R.: 1999, CIR associated energetic particles in the inner and middle heliosphere. Space Sci. Rev. 89, $77-90$.

Mason, G.M., Gold, R.E., Krimigis, S.M., Mazur, J.E., Andrews, G.B., Daley, K.A., Dwyer, J.R., Heuerman, K.F., James, T.L., Kennedy, M.J., et al.: 1998, The Ultra-Low-Energy Isotope Spectrometer (ULEIS) for the ACE spacecraft. Space Sci. Rev. 86, 409-448.

Mason, G.M., Leske, R.A., Desai, M.I., Cohen, C.M.S., Dwyer, J.R., Mazur, J.E., Mewaldt, R.A., Gold, R.E., Krimigis, S.M.: 2008, Abundances and energy spectra of corotating interaction region heavy ions observed during Solar Cycle 23. Astrophys. J. 678, 1458-1470.

Mason, G.M., Desai, M.I., Mall, U., Korth, A., Bucik, R., von Rosenvinge, T.T., Simunac, K.D.: 2009a, In-situ observations of CIRs on STEREO, Wind, and ACE during 2007-2008. Solar Phys. 256, 393-408. 
Mason, G.M., Nitta, N.V., Cohen, C.M.S., Wiedenbeck, M.E.: 2009b, Solar energetic particle ${ }^{3}$ He-rich events from the nearly quiet sun in 2007-2008. Astrophys. J. 700, L56-L59.

McComas, D.J., Ebert, R.W., Elliott, H.A., Goldstein, B.E., Gosling, J.T., Schwadron, N.A., Skoug, R.M.: 2008, Weaker solar wind from the polar coronal holes and the whole Sun. Geophys. Res. Lett. 35, L18103. doi:10.1029/2008GL034896.

Mewaldt, R.A., Cohen, C.M.S., Mason, G.M., Cummings, A.C., Desai, M.I., Leske, R.A., Raines, J., Stone, E.C., Wiedenbeck, M.E., von Rosenvinge, T.T., Zurbuchen, T.H.: 2007, On the differences in composition between solar energetic particles and solar wind. Space Sci. Rev. 130, 207-219.

Ogilvie, K.W., Desch, M.D.: 1997, The Wind spacecraft and its early scientific results. Adv. Space Res. 20, $559-568$.

Pizzo, V.: 1978, A three-dimensional model of corotating streams in the solar wind: 1 . Theoretical foundations. J. Geophys. Res. 83, $5563-5572$.

Reames, D.V.: 1999, Particle acceleration at the Sun and in the heliosphere. Space Sci. Rev. 90, 413-491.

Richardson, I.G.: 2004, Energetic particles and corotating interaction regions in the solar wind. Space Sci. Rev. 111, $267-376$.

Sanderson, T.R., Lin, R.P., Larson, D.E., McCarthy, M.P., Parks, G.K., Bosqued, J.M., Lormant, N., Ogilvie, K., Lepping, R.P., Szabó, A., et al.: 1998, Wind observations of the influence of the Sun's magnetic field on the interplanetary medium at 1 AU. J. Geophys. Res. 103, $17235-17247$.

Schatten, K.H., Wilcox, J.M., Ness, N.F.: 1969, A model of interplanetary and coronal magnetic fields. Solar Phys. 6, $442-455$.

Scholer, M., Hovestadt, D., Klecker, B., Gloeckler, G.: 1979, The composition of energetic particles in corotating events. Astrophys. J. 227, $323-328$.

Schwenn, R.: 1978, Large-scale structure of the interplanetary medium. In: Schwenn, R., Marsch, E. (eds.) Physics of the Inner Heliosphere I, Springer, Berlin, 99-181.

Smith, E.J., Balogh, A.: 2008, Decrease in heliospheric magnetic flux in this solar minimum: recent Ulysses magnetic field observations. Geophys. Res. Lett. 35, L22103. doi:10.1029/2008GL035345.

van Hollebeke, M.A.I., McDonald, F.B., Trainor, J.H., von Rosenvinge, T.T.: 1978, The radial variation of corotating energetic particle streams in the inner and outer solar system. J. Geophys. Res. 83, 47234731.

Wimmer-Schweingruber, R.F., Crooker, N.U., Balogh, A., Bothmer, V., Forsyth, R.J., Gazis, P., Gosling, J.T., Horbury, T., Kilchenmann, A., Richardson, I.G., et al.: 2006, Understanding coronal mass ejection signatures. Space Sci. Rev. 123, $177-216$. 